Article

\title{
Radiation and Magnetic Field Impacts on Time-Dependent Mixed Convection Flow and Heat Transmission of Maxwellian Fluid Past A Stretching Sheet
}

\author{
Sajjad Haider ${ }^{1, *}$, Imran Syed Muhammad ${ }^{2}$, Yun-Zhang Li ${ }^{1}$, Faraz ${ }^{3}$ and Adnan Saeed Butt ${ }^{2}$ \\ 1 College of Applied Science, Beijing University of Technology, Beijing 100124, China; yzlee@bjut.edu.cn \\ 2 Department of Mathematics, Govt postgraduate Gordon College, Rawalpindi 46000, Pakistan; \\ syedsiim@gmail.com (I.S.M.); adnansaeedbutt85@gmail.com (A.S.B.) \\ 3 Department of Applied Mathematics, Northwestern Polytechnical University, Xi'an 710000, China; \\ faraz313pak@mail.nwpu.edu.cn \\ * Correspondence: Sajjadsaleem266@hotmail.com; Tel.: +86-158-1140-5741
}

Received: 17 December 2019; Accepted: 16 February 2020; Published: 27 February 2020

\begin{abstract}
The current study was devoted to explicating the impacts of heat transmission in an unsteady mixed convection flow of an upper convected Maxwell (UCM) fluid passing over a continuously stretching surface under the influence of radiation and magnetic field. Appurtenant similarity transmutations were adopted in order to express the constitutive boundary layer Equations of flow and heat transmission in non-dimensionalized form. The reduced system of partial differential Equations was solved by implementing the implicit finite difference method (IFDM). Our center of attention was to scrutinize the behavior of influential flow parameters on some significant features of flow and heat transmission, which were briefly examined, discussed, and presented in both graphical and tabular formats. Finally, a comparison was established with existing literature in limiting cases to support the present results, and a good agreement was found, corroborating our work. It was predicted that the thermal diffusion rate could be controlled by varying the Prandtl number. Moreover, a rise in radiation and magnetic field parameters reduced the skin friction coefficient and led to enhance the heat transmission rate at the surface. The outcomes of the study might have viable implementations in order to improve the quality of industrial products.
\end{abstract}

Keywords: stretching sheet; Maxwell fluid; heat transfer; implicit finite difference method; mixed convection

\section{Introduction}

The fact-finding concerning the flow and heat transmission over mobile surfaces has remarkable significance regardless of its relevance in various technological processes, such as fiber manufacturing in glass and polymers, metallurgy, glass blowing, paper production, hot rolling, condensation phenomena, fiber spinning, and hot rolling [1]. In all mentioned cases, the most commonly used coolant liquid is water. The quality of end product mainly confides in on heat transmission rate and coefficient of skin friction. Therefore, the flow field and heat transmission studies can be of considerable significance. The pioneering work on the flow of a viscous fluid and heat transmission over a stretchable moving plate was reported by Sakiadis [2], who opened new doors for researchers. Initially, the analytical solution for a flow problem over the contracting sheet was presented by Crane [3]. Later, numerous studies were carried out to extend the research under various aspects for a better explanation of experiments and fluid behaviors [4-10]. 
In recent times, the flow analysis of non-Newtonian fluids has been discussed vastly due to its significance in industrial engineering. Most of the industrial fluids, such as paints, glues, polymers, and printing ink, are non-Newtonian in nature. Due to the complexity, familiar Navier-Stokes Equations cannot exhibit all characteristics of such fluids in an appropriate way. The majority of available models for such fluids are basic, like power laws with grades two or three [11-17]. These models are frequently utilized in fluids modeling, but they cannot foretell the impacts of elasticity. A lot of researchers have introduced the upper convected Maxwell (UCM) fluid model, which anticipates stress relaxation. This rheological model also debars the complicated impacts of shear-dependent viscosity, thus enabling one to concentrate merely on the impacts of fluid elasticity [18-23]. Thermoplastic polymers and various metals in the proximity of their melting point and most of the geo-materials behave as typical Maxwell fluids. The mathematical model of the Maxwell fluid has been employed vastly to characterize the polymeric solutions/fluids and also to express the rheology of crude oil, glycerin, flour dough, toluene, etc.

The study concerning the impacts of thermal radiation and magnetic field on mixed convective flows is a new facet in the context of stretchable surfaces. Radiation effects play a key role in nuclear reactors, space technology, missile re-entry, hypersonic flights, and high-temperature procedures. Thence, many authors have concentrated on this subject as a source of energy transmission, and several attempts are made in order to improve the idea of radiative transfer in such processes. Cess [24] was the first to probe thermal radiation impacts with free convection heat transmission along a vertical plate by employing a perturbation technique. Hossain and Takhar [25] treated a similar problem of a viscous fluid with uniform temperature by utilizing the Keller Box finite difference scheme. Cheng and Ozisik [26] deployed a normal expansion method to treat the radiation effects for an isotropically scattering fluid. Raptis [27] and Hossain et al. [28] explored radiation effects on free convection flow in permeable media. T. Hayat et al. [29] proposed an analytical solution by employing the homotopy analysis method (HAM) to examine the radiation and magnetic field effects over mixed convection stagnation point flow. The impacts of radiation on MHD-Rayleigh flow along with uniform surface heat flux were evaluated by Duwairi and Duwairi [30]. The state-of-the-art is briefly encapsulated in the studies [31-35].

The majority of the aforementioned investigations considered both the flow and temperature fields in a steady state. However, in some instances, including sudden flat sheet stretching or gradual change in its temperature may render the flow field unsteady. Impetuous stretch in the sheet's surface immediately generates an inviscid flow, but it takes a bit longer to develop in the viscous layer close to the sheet. A very few studies have explored the scenarios wherein the stretching force, together with surface temperature, alter with time. Some studies [36-41] employed a similarity method to investigate unsteady stretching surface at constant temperature and derive ordinary differential Equations from the time-dependent boundary layer Equations. Liu and Anderson [42] analyzed the thermal attributes of a liquid film operated by an irregular stretching surface with recommended temperature changes of the stretching sheet. Lately, similarity solutions were obtained for heat transfer as well as unsteady flow across a stretching sheet at varying conditions [43-47]. Bhattacharyya et al. [48] examined the impacts of slip condition on the stagnation point flow of an unsteady boundary layer across a stretching sheet.

It is perceived that a lot of work has been done on UCM fluids, and numerous methods are introduced to compute the specific type of problems. However, no efforts have been made yet to find the solution of heat transmission of an unsteady mixed convection flow of Maxwellian fluids past a continuing stretched sheet in the presence of radiation and magnetic field effects. The focal point of the ongoing study was to fill-up this gap in the existing literature. The exact solution was computed, and further implicit finite difference method (IFDM) method was implemented in order to get the solution to the designed problem. In addition, graphical results were illustrated and analyzed. 


\section{Problem Development}

Let us take into account a two dimensional (2-D) unsteady laminar flow of Maxwellian fluid past over a surface stretching continuously with velocity $U_{w}(x)$ in the plane $y=0$. The $x$-axis is taken along the sheet, and the $y$-axis is normal to the surface. We presume that $U_{w}(x)$ varies linearly, while surface temperature $T_{w}(x)$ has a quadratic variation with the distance $x$ measured from $\mathrm{O}$. The flow geometry is presented in Figure 1. Keeping in view the above-stated assumptions, the fluid flow can be described by the following Equations. The boundary layer flow model and concentration field are presented as [49].

$$
\begin{gathered}
\frac{\partial u}{\partial x}+\frac{\partial v}{\partial y}=0 \\
\frac{\partial u}{\partial t}+u \frac{\partial u}{\partial x}+v \frac{\partial u}{\partial y}+\lambda^{*}\left[u^{2} \frac{\partial^{2} u}{\partial x^{2}}+v^{2} \frac{\partial^{2} u}{\partial y^{2}}+2 u v \frac{\partial^{2} u}{\partial x \partial y}\right]=v \frac{\partial^{2} u}{\partial y^{2}} \\
-\frac{\sigma B_{0}^{2}}{\rho} u+g \beta^{*}\left(T-T_{\infty}\right), \\
\frac{\partial T}{\partial t}+u \frac{\partial T}{\partial x}+v \frac{\partial T}{\partial y}=\frac{\alpha}{\rho c_{p}} \frac{\partial^{2} T}{\partial y^{2}}-\frac{1}{\rho c_{p}} \frac{\partial q}{\partial y}
\end{gathered}
$$

The suitable boundary constraints for the problem can be written as

$$
\begin{aligned}
& u=U_{w}(x), \quad v=0, \quad T=T_{w}(x)=T_{\infty}+\Delta T(x) \quad \text { at } y=0, \\
& u \rightarrow 0, \quad T \rightarrow T_{\infty} \quad \text { as } y \rightarrow \infty .
\end{aligned}
$$

where $u$ and $v$ are the velocity components in the $\mathrm{x}$ and $\mathrm{y}$ directions, respectively, $\lambda$ is the relaxation time, $T$ is the fluid temperature inside the boundary layer, $t$ is time, $\rho$ is the fluid density, $c_{p}$ is the specific heat capacity at constant pressure of the fluid, where $B_{0}$ is the strength of the magnetic field, $q_{r}$ is the radiative heat flux, $\alpha$ and $v$ are the thermal diffusivity and the kinematic viscosity, respectively.

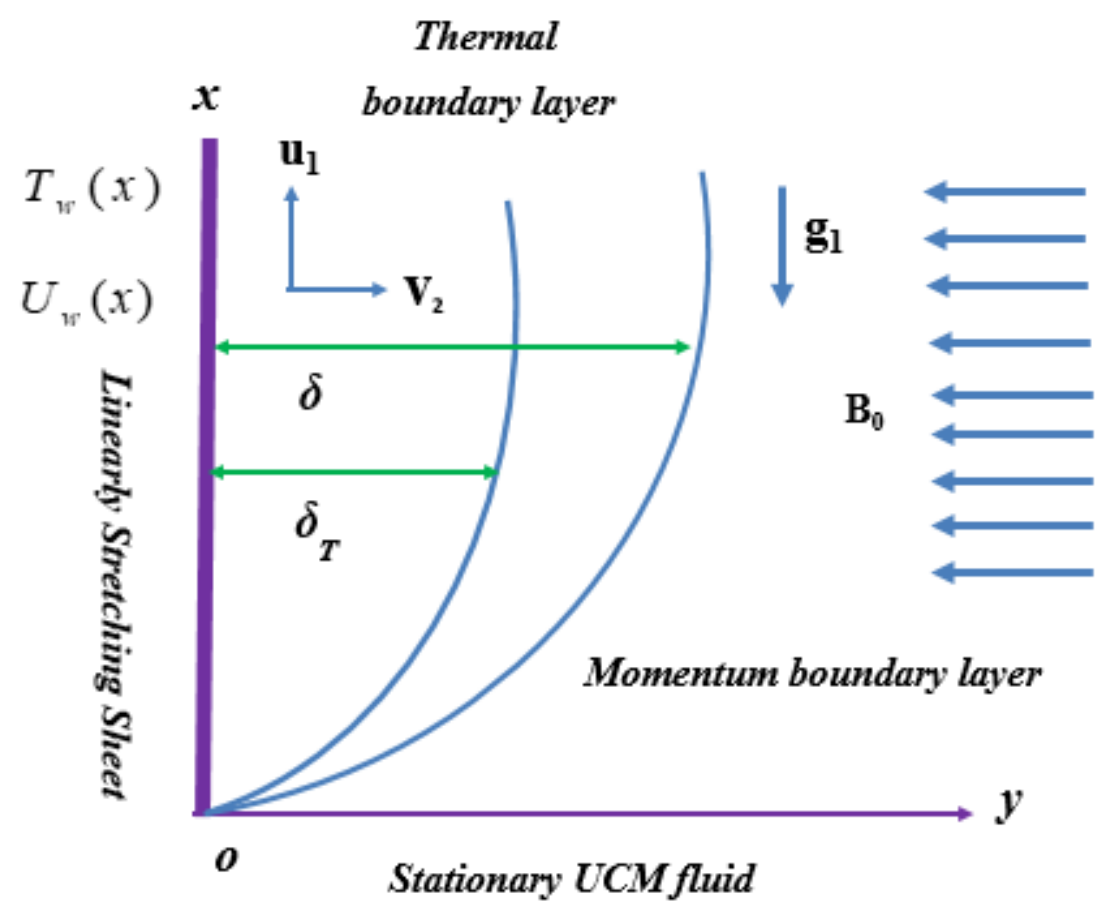

Figure 1. The flow diagram and coordinate system.

We consider the following form of stretching velocity of the surface and temperature

$$
U_{w}(x)=a x, \quad \Delta T(x)=b x^{2}
$$


Making use of the Rosseland approximation for radiation for an optically thick layer [50], we have

$$
q_{r}=-\frac{4 \sigma^{*}}{3 k^{*}} \frac{\partial T^{4}}{\partial y}
$$

where $\sigma^{*}$ and $k^{*}$ denote Stefan-Boltzmann constant and mean absorption coefficient, respectively. We can express the term $T^{4}$ as a linear function of temperature. It is recognized by expanding $T^{4}$ in a Taylor series about $T_{\infty}$ and neglecting higher terms, then one can write

$$
T^{4} \cong 4 T_{\infty}^{3} T-3 T_{\infty}^{4} .
$$

From Equations (3), (6), and (7), one obtains

$$
\frac{\partial T}{\partial t}+u \frac{\partial T}{\partial x}+v \frac{\partial T}{\partial y}=\frac{\alpha}{\rho c_{p}} \frac{\partial^{2} T}{\partial y^{2}}+\frac{16 \sigma^{*} T_{\infty}^{3}}{3 \rho c_{p} k^{*}} \frac{\partial^{2} T}{\partial y^{2}} .
$$

Employing the non-dimensionalized quatities:

$$
\psi=x \sqrt{a v \xi} f(\eta, \xi), \quad \eta=y \sqrt{\frac{a}{v \xi}}, \quad \xi=1-\mathrm{e}^{\tau}, \tau=a t, \theta=\frac{T-T_{\infty}}{\Delta T(x)},
$$

Here, $\tau$, represents dimensionless time, $a>0$, and $\psi$ denotes stream function as $u=\partial \psi / \partial y$, and $v=-\partial \psi / \partial x$, continuity Equation is automatically satisfied as a consequence, and the corresponding other concentration Equations are transmuted as follow:

$$
\begin{gathered}
f^{\prime \prime \prime}+\xi\left(f f^{\prime \prime}-f^{\prime 2}-M f^{\prime}\right)+\beta \xi\left(2 f f^{\prime} f^{\prime \prime}-f^{2} f^{\prime \prime \prime}\right)+\frac{1}{2} \eta(1-\xi) f^{\prime \prime}+\xi \lambda=\xi(1-\xi) \frac{\partial f^{\prime}}{\partial \xi}, \\
\frac{1}{\operatorname{Pr}}\left(1+\frac{4}{3} R_{d}\right) \theta^{\prime \prime}+\xi\left(f \theta^{\prime}-2 f^{\prime} \theta\right)+\frac{1}{2} \eta(1-\xi) \theta^{\prime}=\xi(1-\xi) \frac{\partial \theta}{\partial \xi}
\end{gathered}
$$

Suitable boundary constraints are:

$$
\begin{aligned}
f^{\prime}(0, \xi)=1, f(0, \xi)= & 0, \theta(0, \xi)=1, \\
& \theta(\infty, \xi)=0, f^{\prime}(\infty, \xi)=0,
\end{aligned}
$$

Here, $\beta=a \lambda^{*}, M=\frac{\sigma B_{0}^{2}}{a \rho}$, and $\lambda=\frac{G r_{x}}{\operatorname{Re}_{x}^{2}}$ are the relaxation time, magnetic field parameter, and mixed convection parameter, respectively. The local Grashof number $G r_{x}$, the local Reynold number $\operatorname{Re}_{x}$, the Prandtl number $\operatorname{Pr}$, and the radiation parameter $R_{d}$ are, respectively, defined as $G r_{x}=\frac{g \beta^{*}\left(T-T_{\infty}\right) x^{3}}{v^{2}}$, $\operatorname{Re}_{x}=\frac{U_{w} x}{v}, \operatorname{Pr}=\frac{v}{\alpha}$, and $R_{d}=\frac{4 \sigma^{*} T_{\infty}^{3}}{\alpha k^{*}}$.

The physical parameters $C_{f}$ and $N u$ are defined as:

$$
C_{f}=\frac{\tau_{w}(x)}{\rho\left(U_{w}(x)\right)^{2}} \text { and } N u_{x}=\frac{q_{w}(x) x}{\alpha \Delta T(x)}
$$

where the wall share stress $\tau_{w}$, the heat transfer, and mass transfer are given by $q_{w}$ as:

$$
\tau_{w}(x)=\mu\left(\frac{\partial u}{\partial y}\right)_{y=0} \text { and } q_{w}(x)=-\left(\left(\alpha+\frac{16 \sigma^{*} T_{\infty}^{3}}{3 k^{*}}\right) \frac{\partial T}{\partial y}\right)_{y=0}
$$

By utilizing similarity transformations (5), skin friction coefficient $C_{f}$ and Nusselt number $N u$ can be expressed as:

$$
\xi^{\frac{1}{2}} \operatorname{Re}_{x}^{\frac{1}{2}} C_{f}=f^{\prime \prime}(0, \xi)
$$




$$
\xi^{-\frac{1}{2}} R e_{x}^{-\frac{1}{2}} N u_{x}=-\left(1+\frac{4}{3} R_{d}\right) \theta^{\prime}(0, \xi) .
$$

The Equations (10)-(12) authorize reductions to ODE systems of the governing profiles $f^{\prime}(\eta)$ and $\theta(\eta)$ in the initial unsteady-state flow at $\xi=0$, for a small time and final steady-state flow at large times given by $\xi=1$.

\section{Exact Solution}

\subsection{Initial Steady Flow}

As $\xi=0(\tau=0)$ and also $R_{d}=0$, the Equations (10) and (11) reduces to:

$$
\begin{gathered}
f^{\prime \prime \prime}+(1 / 2) \eta f^{\prime \prime}=0, \\
\frac{1}{\operatorname{Pr}} \theta^{\prime \prime}+(1 / 2) \eta \theta^{\prime}=0,
\end{gathered}
$$

With boundary constraints

$$
\begin{aligned}
f^{\prime}(0,0)=1, f(0,0)= & 0, \theta(0,0)=1, \\
& f(\infty, 0)=0, \theta(\infty, 0)=0 .
\end{aligned}
$$

The analytical results for the Equations (13) and (14) are specified as:

$$
\begin{gathered}
f(\eta, 0)=\eta+\frac{2}{\sqrt{\pi}}\left(1-\mathrm{e}^{\left(-\eta^{2} / 4\right)}\right)-\eta \operatorname{erf}\left(\frac{\eta}{2}\right), \\
\theta(\eta, 0)=1-\frac{1}{\sqrt{\operatorname{Pr}}} \operatorname{erf}\left(\frac{\operatorname{Pr} \eta}{2}\right),
\end{gathered}
$$

The error function $\operatorname{Erf}(z)$ is defined as:

$$
\operatorname{Erf}(z)=\frac{2}{\sqrt{\pi}} \int_{0}^{z} \mathrm{e}^{\left(-t^{2}\right)} d t .
$$

\subsection{Final Unsteady State Flow}

For $\xi=1(\tau \rightarrow \infty)$, corresponding Equations (10) and (11) are reduced to

$$
\begin{gathered}
f^{\prime \prime \prime}+\lambda \theta+\beta f\left(2 f^{\prime} f^{\prime \prime}-f f^{\prime \prime \prime}\right)+\left(f f^{\prime \prime}-f^{\prime 2}-M f^{\prime}\right)=0, \\
\frac{1}{\operatorname{Pr}}\left(1+\frac{4}{3} R_{d}\right) \theta^{\prime \prime}-\left(2 f^{\prime} \theta-f \theta^{\prime}\right)=0,
\end{gathered}
$$

Suitable boundary constraints

$$
\begin{aligned}
f^{\prime}(0,1)=1, f(0,1)=0, \theta(0,1)=1, \\
f^{\prime}(\infty, 1)=0, \theta(\infty, 1)=0,
\end{aligned}
$$

Equation (23) affirms the exact results at $\beta=0$ and $\lambda=0$ as:

$$
f(\eta, 1)=\frac{1}{b}\left(1-\mathrm{e}^{(-b \eta)}\right),
$$

as $b=\sqrt{1+M}$. Assume $c=\left(1+\frac{4}{3} R_{d}\right)$. 
Invoking Equation (25) into Equations (23) and (24) and familiarizing the change $\xi=-\frac{\operatorname{Pr}}{c} e^{-b \eta}$, shown as:

$$
\begin{gathered}
b^{2} \xi \frac{d^{2} \theta}{d \xi^{2}}+\left(b^{2}-\frac{\operatorname{Pr}}{c}-\xi\right) \frac{d \theta}{d \xi}+2 \theta=0 \\
\theta\left(-\frac{\operatorname{Pr}}{c}\right)=1, \theta^{\prime}(0)=0 .
\end{gathered}
$$

Equation (26) may be changed in the Confluent hypergeometric "Kummer's function", and the results are satisfying the Equations (23) and (24) as

$$
“ \theta(\eta, 1)=\frac{\text { Kummer }\left[\left(\frac{2 b^{2} c+\operatorname{Pr}}{b^{2} c}\right),\left(\frac{b^{2} c+\operatorname{Pr}}{b^{2} c}\right),\left(\frac{-\operatorname{Pr} e^{-b \eta}}{b^{2} c}\right)\right]\left(-\frac{\operatorname{Pr}}{c} e^{-b \eta}\right)^{\frac{\operatorname{Pr}}{b^{2} c}}}{\text { Kummer }\left[\left(\frac{-2 b^{2} c-\operatorname{Pr}}{b^{2} c}\right),\left(\frac{b^{2} c+\operatorname{Pr}}{b^{2} c}\right),\left(\frac{-\operatorname{Pr}}{b^{2} c}\right)\right]\left(-\frac{\operatorname{Pr}}{c}\right)^{\frac{\operatorname{Pr}}{b^{2} c}}} " .
$$

\section{Implicit Finite Difference Method (IFDM)}

We recast Equations (10) and (11) as a set of simultaneous Equations by introducing the variables $U, V, P$ :

$$
\begin{gathered}
f^{\prime}=U, f^{\prime \prime}=V, \theta=W, \theta^{\prime}=P . \\
V^{\prime}+\xi\left(f V-U^{2}-M U\right)+\beta \xi\left[2 f U V-f^{2} V\right]+\frac{1}{2} \eta(1-\xi) V+\xi \lambda W=\xi(1-\xi) \frac{\partial U}{\partial \xi} \\
\frac{1}{\operatorname{Pr}}\left(1+\frac{4}{3} R_{d}\right) P^{\prime}+\frac{1}{2} \eta(1-\xi) P+\xi(f P-2 U \theta)=\xi(1-\xi) \frac{\partial \theta}{\partial \xi^{\prime}} \\
f(0, \xi)=0, U(0, \xi)=1, U(\infty, \xi)=0, \\
\theta(0, \xi)=1, \theta(\infty, \xi)=0 .
\end{gathered}
$$

We now place a net on the $(\eta, \xi)$-plane defined by

$$
\begin{aligned}
& \eta_{0}=0, \eta_{j}=\eta_{j-1}+h_{j}, j=1,2,3, \ldots, J, \eta_{J}=\eta_{\infty}, \\
& \xi^{0}=0, \xi^{n}=\xi^{n-1}+k^{n}, n=1,2,3, \ldots
\end{aligned}
$$

If $g_{j}^{n}$ denotes the value of any variable at $\left(\eta_{j}, \xi^{n}\right)$, then the variables and derivatives of Equations (27) and (28) at $\left(\eta_{j-1 / 2}, \xi^{n-1 / 2}\right)$ are replaced by

$$
\begin{gathered}
g_{j-1 / 2}^{n-1 / 2}=\frac{1}{4}\left(g_{j}^{n}+g_{j-1}^{n}+g_{j}^{n-1}+g_{j-1}^{n-1}\right), \\
\left(\frac{\partial g}{\partial \eta}\right)_{j-1 / 2}^{n-1 / 2}=\frac{1}{2 h_{j}}\left(g_{j}^{n}-g_{j-1}^{n}+g_{j}^{n-1}-g_{j-1}^{n-1}\right), \\
\left(\frac{\partial g}{\partial \xi}\right)_{j-1 / 2}^{n-1 / 2}=\frac{1}{2 k^{n}}\left(g_{j}^{n}-g_{j}^{n-1}+g_{j-1}^{n}-g_{j-1}^{n-1}\right),
\end{gathered}
$$

We now show the finite-difference approximation of Equations (31) and (32) for the mid-point $\left(\eta_{j-1 / 2}, \xi^{n}\right)$, as below

$$
\begin{gathered}
h_{j}^{-1}\left(f_{j}^{n}-f_{j-1}^{n}\right)=U_{j-1 / 2^{\prime}}^{n} \\
h_{j}^{-1}\left(U_{j}^{n}-U_{j-1}^{n}\right)=V_{j-1 / 2^{\prime}}^{n} \\
h_{j}^{-1}\left(V_{j}^{n}-V_{j-1}^{n}\right)-\frac{\beta \xi^{n-1 / 2}}{2}\left[\begin{array}{l}
\left.\left(f^{2}\right)_{j-1 / 2}^{n} h_{j}^{-1}\left(V_{j}^{n}-V_{j-1}^{n}\right)+\left(f^{2}\right)_{j-1 / 2}^{n} h_{j}^{-1}\left(V_{j}^{n-1}-V_{j-1}^{n-1}\right)+\right] \\
\left(f^{2}\right)_{j-1 / 2}^{n-1} h_{j}^{-1}\left(V_{j}^{n}-V_{j-1}^{n}\right)+4 \xi^{n-1 / 2}(f U V)_{j-1 / 2}^{n}
\end{array}\right] \\
+\xi^{n-1 / 2}\left[(f V)_{j-1 / 2}^{n}-\left(U^{2}\right)_{j-1 / 2}^{n}\right]-\xi^{n-1 / 2} M U_{j-1 / 2}^{n}+\frac{\eta}{2}\left(1-\xi^{n-1 / 2}\right) V_{j-1 / 2}^{n}-2 \alpha_{n}\left(1-\alpha_{n}\right) U_{j-1 / 2}^{n} \\
=R_{j-1 / 2}^{n-1 / 2},
\end{gathered}
$$




$$
\begin{aligned}
& h_{j}^{-1}\left(\theta_{j}^{n}-\theta_{j-1}^{n}\right)=P_{j-1 / 2}^{n} \\
& \frac{1}{\operatorname{Pr}}\left(1+\frac{4}{3} R_{d}\right) h_{j}^{-1}\left(P_{j}^{n}-P_{j-1}^{n}\right)++\frac{\eta}{2}\left(1-\xi^{n-1 / 2}\right) P_{j-1 / 2}^{n}+\xi^{n-1 / 2}\left[(f P)_{j-1 / 2}^{n}-2(U \theta)_{j-1 / 2}^{n}\right]-2 \alpha_{n}\left(1-\alpha_{n}\right) \theta_{j-1 / 2}^{n} \\
& =T_{j-1 / 2}^{n-1} \text {, }
\end{aligned}
$$

where

$$
\begin{aligned}
& \alpha_{n}=\xi^{n-1 / 2} / k^{n}, \\
& R_{j-1 / 2}^{n-1}=-L_{j-1 / 2}^{n-1}+2 \alpha_{n}\left(1-\alpha_{n}\right) U_{j-1 / 2^{\prime}}^{n-1} \\
& L_{j-1 / 2}^{n-1}=h_{j}^{-1}\left(V_{j}^{n-1}-V_{j-1}^{n-1}\right)+\frac{\xi^{n-1 / 2}}{2}\left[2(f U V)_{j-1 / 2}^{n-1}-\left(f^{2}\right)_{j-1 / 2}^{n-1} h_{j}^{-1}\left(V_{j}^{n-1}-V_{j-1}^{n-1}\right)\right]+\xi^{n-1 / 2}\left[(f V)_{j-1 / 2}^{n-1}-\left(U^{2}\right)_{j-1 / 2}^{n-1}\right] \\
& -M \xi^{n-1 / 2} U_{j-1 / 2}^{n-1}+\frac{\eta}{2}\left(1-\xi^{n-1 / 2}\right) V_{j-1 / 2^{\prime}}^{n-1} \\
& T_{j-1 / 2}^{n-1}=-M_{j-1 / 2}^{n-1}+2 \alpha_{n}\left(1-\alpha_{n}\right) \theta_{j-1 / 2^{\prime}}^{n-1} \\
& M_{j-1 / 2}^{n-1}=\frac{1}{\operatorname{Pr}}\left(1+\frac{4}{3} R_{d}\right) h_{j}^{-1}\left(P_{j}^{n-1}-P_{j-1}^{n-1}\right)+\xi^{n-1 / 2}\left[(f P)_{j-1 / 2}^{n-1}-2(U \theta)_{j-1 / 2}^{n-1}\right]+\frac{\eta}{2}\left(1-\xi^{n-1 / 2}\right) P_{j-1 / 2}^{n} .
\end{aligned}
$$

The boundary constraints are

$$
f_{0}^{n}=0, U_{0}^{n}=1, \theta_{0}^{n}=1, U_{J}^{n}=0, \theta_{J}^{n}=0 .
$$

If $f_{j}^{n-1}, U_{j}^{n-1}, V_{j}^{n-1}, \theta_{j}^{n-1}, P_{j}^{n-1}$ are presumed to be well- known for $0 \leq j \leq J$, Equations (36)-(40) form a system of $5 J+5$. Equations for the solution of $5 J+5$ have unknowns $f_{j}^{n}, U_{j}^{n}, V_{j}^{n}, \theta_{j}^{n}, P_{j}^{n}, j=$ $0,1,2, \ldots, J$. Newton's linearization method is implemented to linearize this system of Equations, and Equations are solved by employing the Keller-box elimination scheme. The procedure is initiated with $\xi=0$, and we begin with a set-up of estimated unconditionally convergent profiles for $f, U, V, \theta$, and $P$, which are exerted in the Keller-box scheme along with second-order precision to advance gradually along the boundary-layer. In order to compute these functions, the difference in procedure is taken less than $10^{-7}$, i.e., $\left|\delta f^{i}\right| \leq 10^{-7}$, where the superscript $i$ denotes the number of iterations. To achieve speedy convergence, we take $\eta_{j}=\sinh (j / a)$ in order to save calculations time and space.

\section{Numerical Results and Discussions}

Heat transmission of unsteady mixed convection flow of an upper convected Maxwellian fluid past a continuously stretching surface under the influence of radiation and magnetic field impacts was studied. Exact solutions for initial steady and final unsteady state flows were attained, and, finally, the implicit finite difference scheme was utilized to solve the designed problem. In order to examine the flow behavior of Maxwell fluid for velocity and temperature profiles, a rigorous numerical computation was accomplished for numerous values of parameters, which narrated the flow characteristics. Moreover, Table 1 is presented to establish a comparison with existing literature (Abel et al. (2012) [23]) and Sadeghy et al. (2006) [19]) in order to support the results of the proposed study, and a good agreement was found, corroborating our work. Table 2 shows the values of $f^{\prime \prime}(0, \xi)$ and $-\theta^{\prime}(0, \xi)$ for different values of flow parameters.

Table 1. Comparison of $f^{\prime \prime}(0)$ for various values of $\beta$ and $M$ when $\lambda=0$ and $\xi=1$.

\begin{tabular}{cccccc}
\hline & Sadeghy et al. [19] & Subhas et al. [23] & Present Results & Subhas et al. [21] & Present Results \\
\cline { 2 - 6 } & \multicolumn{3}{c}{$\boldsymbol{M}=0.2$} \\
\hline 0.0 & -1.0000000 & -0.999962 & -0.999962 & -1.095445 & -1.095151 \\
0.2 & -1.0549000 & -1.051948 & -1.052001 & -1.188270 & -1.188321 \\
0.4 & -1.1008400 & -1.101850 & -1.101843 & -1.275878 & -1.275888 \\
0.6 & -1.0015016 & -1.150163 & -1.150133 & -1.358733 & -1.358771 \\
0.8 & -1.1987200 & -1.196692 & -1.196634 & -1.437369 & -1.437357 \\
1.2 & - & -1.285257 & -1.285269 & -1.512280 & -1.512280 \\
1.6 & - & -1.368641 & -1.368630 & -1.095445 & -1.095445 \\
2.0 & - & -1.447617 & -1.447654 & -1.188270 & -1.188119 \\
\hline
\end{tabular}


Table 2. $-\theta^{\prime}(0, \xi)$ and $f^{\prime \prime}(0, \xi)$ exact solutions found through the different procedure for the $\xi$ as $\operatorname{Pr}=1, M=0.5, R_{d}=0.5, \lambda=1.0$, and $\beta=0.5$.

\begin{tabular}{ccc}
\hline$\xi$ & $f^{\prime \prime}(\mathbf{0}, \boldsymbol{\xi})$ & $-\boldsymbol{\theta}^{\prime}(\mathbf{0}, \boldsymbol{\xi})$ \\
\hline 0.0 & -0.56784 & 0.86725 \\
0.1 & -0.59342 & 0.97241 \\
0.2 & -0.61735 & 1.06797 \\
0.3 & -0.64177 & 1.16383 \\
0.4 & -0.66621 & 1.25733 \\
0.5 & -0.69096 & 1.35039 \\
0.6 & -0.71573 & 1.44164 \\
0.7 & -0.74067 & 1.53248 \\
0.8 & -0.76554 & 1.62206 \\
0.9 & -0.79034 & 1.71184 \\
1.0 & -0.81459 & 1.80201 \\
\hline
\end{tabular}

Impacts of relaxation time parameter $\beta$ on skin friction and heat transfer rate are displayed in Figure $2 a, b$. A decline in skin friction and the heat transfer rate was observed with the rise in values of $\beta$.
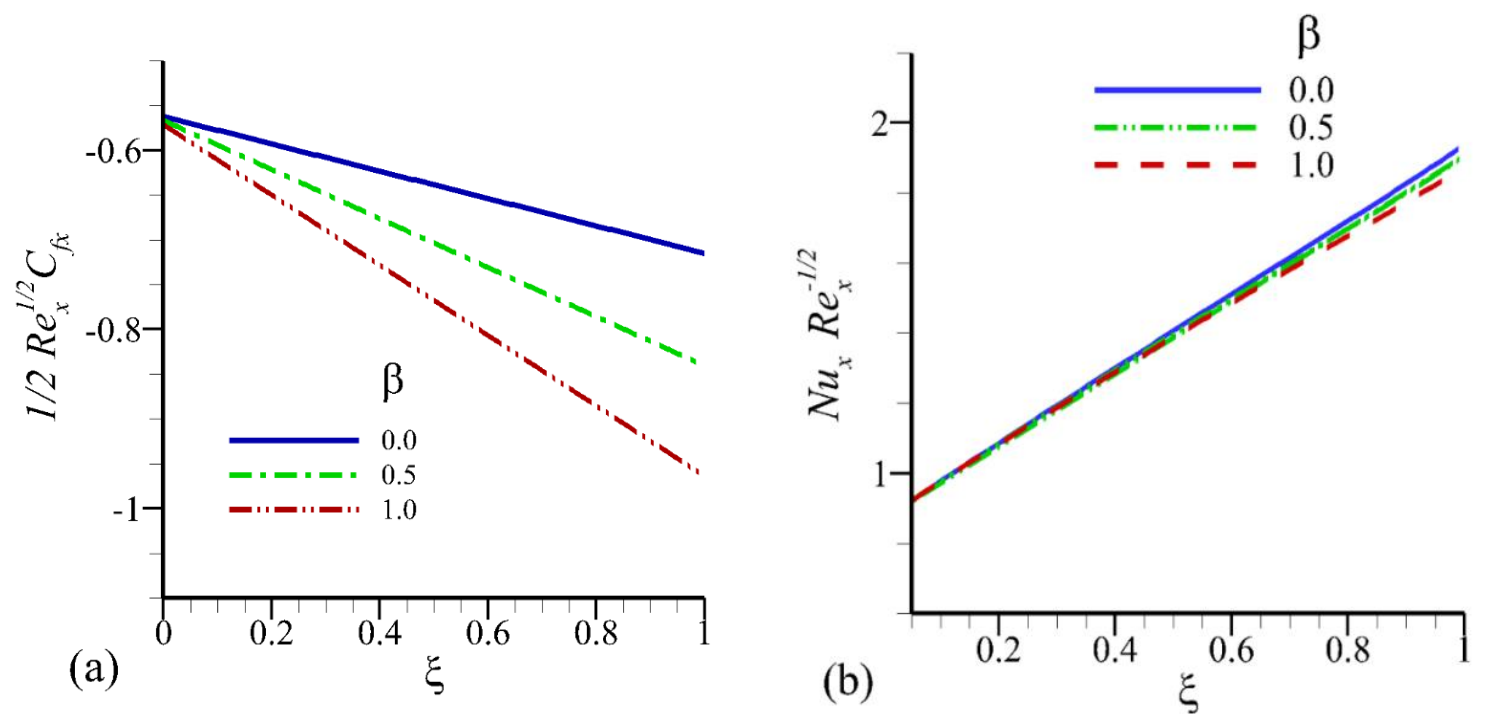

Figure 2. Graph of (a) $\frac{1}{2} R e_{x}^{1 / 2} C_{f x}$, (b) $N u_{x} R e_{x}^{-1 / 2}$ with $\xi$ for changing values of $\beta$ while $\operatorname{Pr}=\lambda=1.0$ and $R_{d}=M=0.5$.

Figure $3 a, b$ exhibit the influence of different mixed convection parameter $\lambda$ on skin friction and heat transfer rate. These figures narrate that both $\frac{1}{2} R e_{x}^{1 / 2} C_{f}$ and $N u_{x} R e_{x}^{-1 / 2}$ amplified with the rise in values of $\xi$ and $\lambda$. It seemed quite logical that due to boost in buoyancy impact, velocity had to rise, and thus, as a consequence, the wall shear stress increased. When $\xi=0$, Equations (10) and (11) had an exact solution in the form of Equations (25) and (28), assisting us to validate our numerical results. 

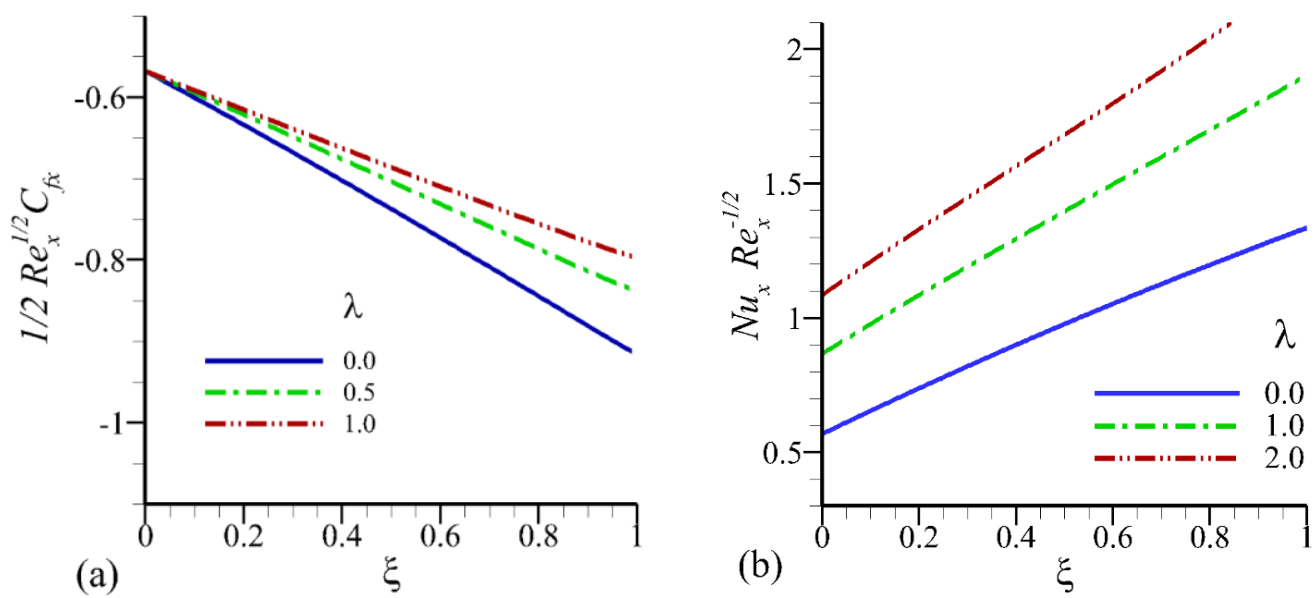

Figure 3. Graph of (a) $\frac{1}{2} R e_{x}^{1 / 2} C_{f x}$, (b) $N u_{x} R e_{x}^{-1 / 2}$ with $\xi$ for changed values of $\lambda$ while $R_{d}=M=\beta=0.5$, and $\operatorname{Pr}=1.0$.

From Figure 4a,b, it could be clearly predicted that an enhancement in the radiation parameter $R_{d}$ led to reducing the coefficient of local skin friction and increased the heat transmission rate at the surface. This phenomenon could be recognized from the fact that due to the rise in radiation parameter $R_{d}$, the ambient fluid temperature decreased, and Roseland's mean absorption coefficient $k$ increased, diminishing the skin friction and augmenting the rate of heat transmission at the surface.
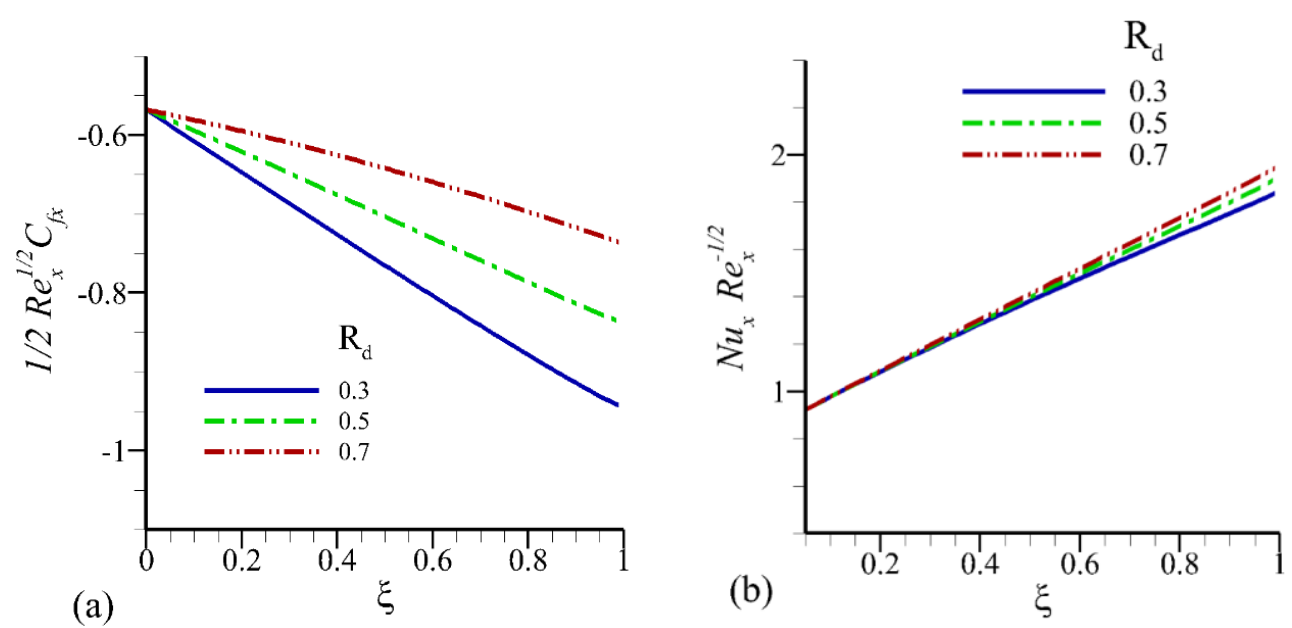

Figure 4. Graph of (a) $\frac{1}{2} R e_{x}^{1 / 2} C_{f x}$, (b) $N u_{x} R e_{x}^{-1 / 2}$ with $\xi$ for changed values of $R_{d}$ while $M=\beta=0.5$ and $\operatorname{Pr}=\lambda=1.0$.

Variations in skin friction and heat transfer against different values of $\xi$ for different Prandtl number Pr are sketched in Figure 5a,b. Figure 5 a indicates a reduction in skin friction, while Figure $5 b$ shows an elevation in heat transmission rate with rising values of $\xi$. It was deduced that buoyancy forces got higher as Pr became smaller, elevating the fluid pressure. Therefore, velocity enhanced in the neighborhood of heated surface, and hence a boost in skin friction was witnessed. Moreover, we observed that, for lower Pr values, the influence of the warmed surface was intense within the fluid; thus, there was a lower Nusselt number. 

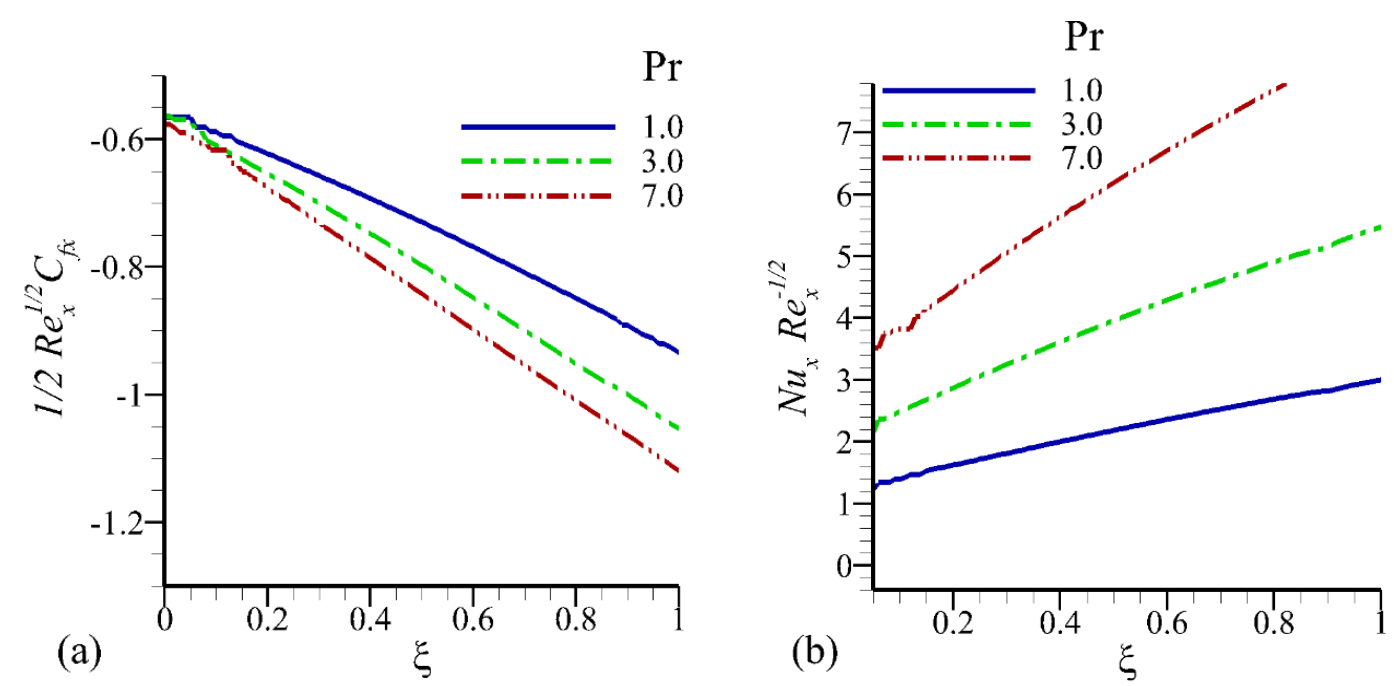

Figure 5. Graph of (a) $\frac{1}{2} R e_{x}^{1 / 2} C_{f_{x}},(\mathbf{b}) N u_{x} R e_{x}^{-1 / 2}$ with $\xi$ for changed values of Pr while $R_{d}=M=\beta=0.5$, and $\lambda=1.0$.

It is evident from Figure 6 that an increase in the magnetic field parameter $M$ turned down the local skin friction. This was due to the fact that with the elevation of $M$, the Lorentz force became stronger and retarded the flow, and the coefficient of local skin friction reduced consequently.

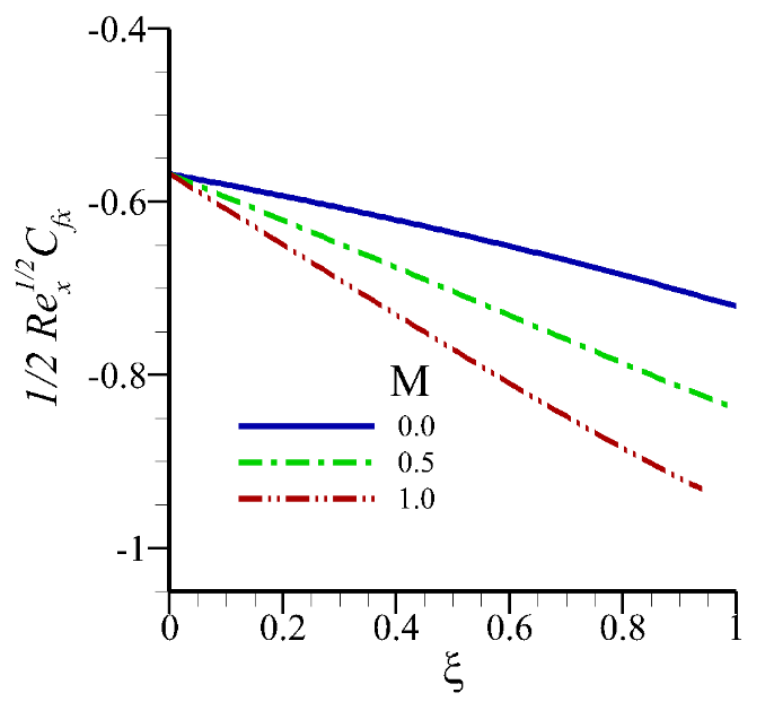

Figure 6. Graph of $\frac{1}{2} R e_{x}^{1 / 2} C_{f_{x}}$ with $\xi$ for changed values of $M$ while $\operatorname{Pr}=\lambda=1.0$, and $R_{d}=0.5$.

Figure $7 \mathrm{a}, \mathrm{b}$ unveil the impact of different $\operatorname{Pr}$ values on $f^{\prime}(\eta, \xi)$ and $\theta(\eta, \xi)$ with variation in $\xi$. Figure 7a discloses a velocity rise in the nearby region of the heated surface due to high fluid conductivity for smaller Pr values. It was because of high fluid conductivity, causing significant density differences and, consequently, a high buoyancy force. Figure $7 \mathrm{~b}$ indicates a decline in temperature distribution with a rise in $\mathrm{Pr}$, as higher values of Pr slowed down the thermal diffusion. 

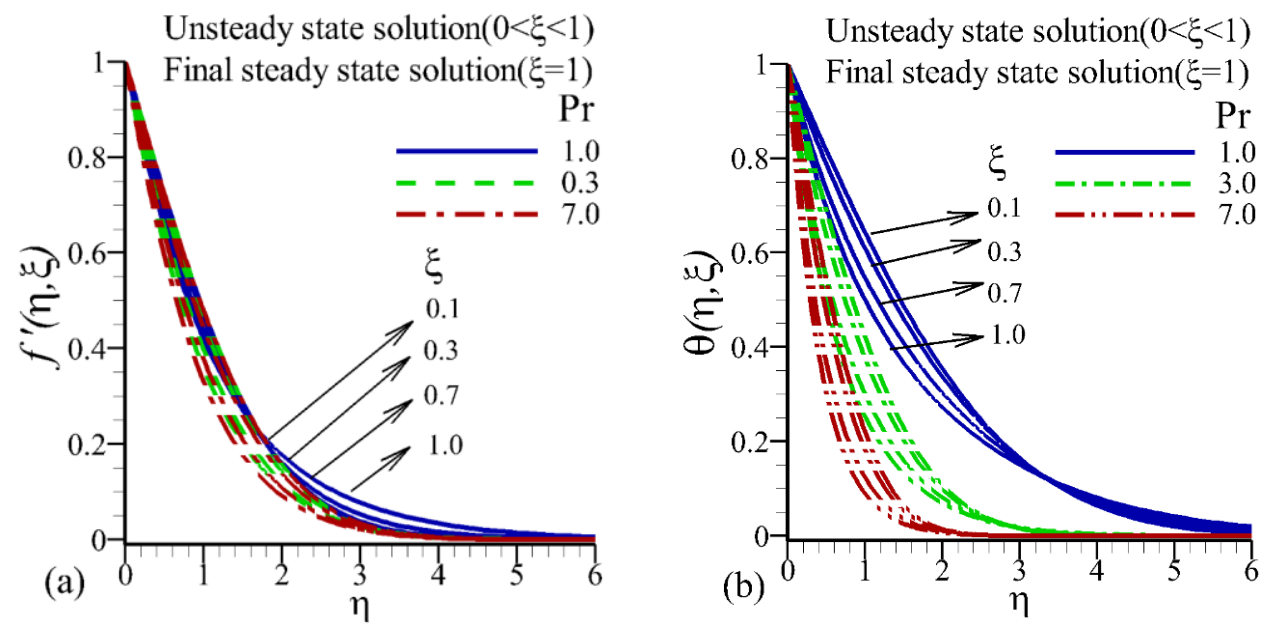

Figure 7. (a) Velocity $f^{\prime}(\eta, \xi)$, (b) Temperature $\theta(\eta, \xi)$ against $\eta$ when $\operatorname{Pr}=1.0,3.0$, and $7.0, \xi=$ $0.1,0.3,0.7,1.0$, while $R_{d}=M=\beta=0.5$ and $\lambda=1.0$.

The impacts of mixed convection parameter $\lambda$ for various values of $\xi$ on velocity and temperature profiles are sketched in Figure 8a,b. Since we knew that $\lambda=0$ represents the case of forced convection and rise in $\lambda$ intensifies the buoyancy force, it resulted in a rise in fluid velocity and the decline in temperature in the neighborhood of surface.
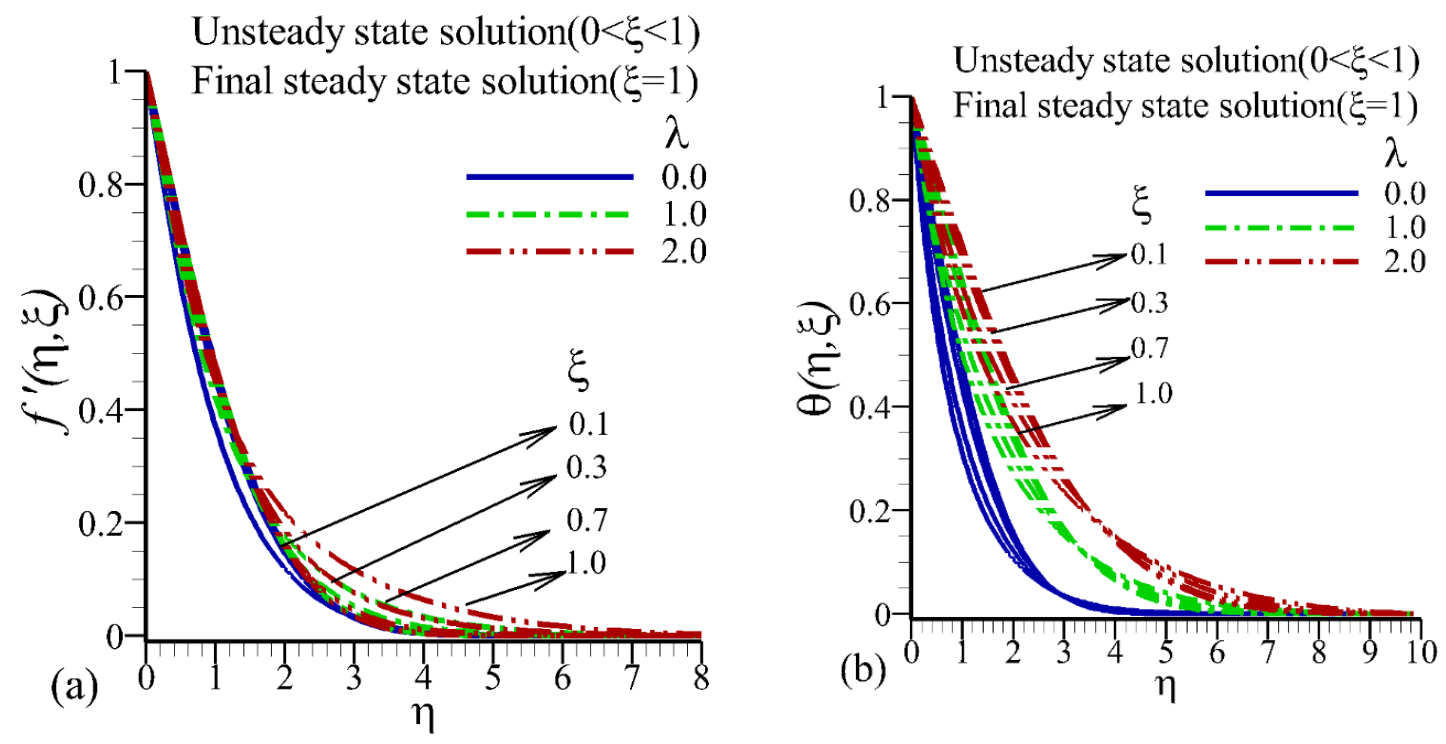

Figure 8. (a) Velocity $f^{\prime}(\eta, \xi)$, (b) Temperature $\theta(\eta, \xi)$ against $\eta$ for $\lambda=0.0,1.0$, and 2.0, $\xi=$ $0.1,0.3,0.7,1.0$, while $R_{d}=M=\beta=0.5$ and $\operatorname{Pr}=1.0$.

Figure 9a presents the effects of the magnetic field parameter $M$ on velocity and temperature profiles. It was obvious that with the rise in values of $M$, the Lorentz force became stronger and retarded the flow of fluid. Figure $9 \mathrm{~b}$ illustrates the variation in the magnetic parameter $M$ on the temperature profile $\theta(\eta, \xi)$. It showed that the magnetic-field parameter had an increasing effect on the thermal boundary layer thickness. In Figure 10a,b, it is shown that by increasing the radiation parameter $R_{d}$, and by keeping the other parameters constant, the velocity and temperature distribution was diminished. 

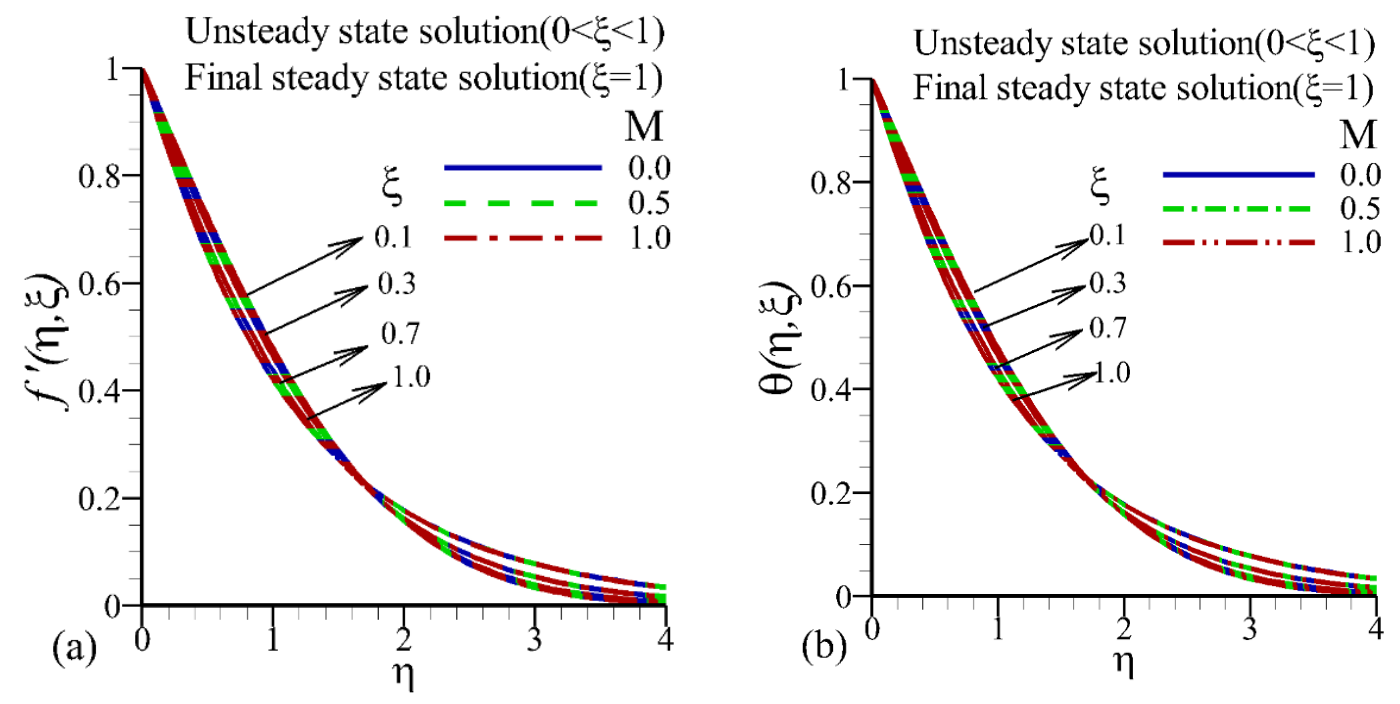

Figure 9. (a) Velocity $f^{\prime}(\eta, \xi),(\mathbf{b})$ Temperature $\theta(\eta, \xi)$ against $\eta$ for $M=0.0,1.0$, and $2.0, \xi=$ $0.1,0.3,0.7,1.0$, while $R_{d}=\beta=0.5$ and $\operatorname{Pr}=\lambda=1.0$.
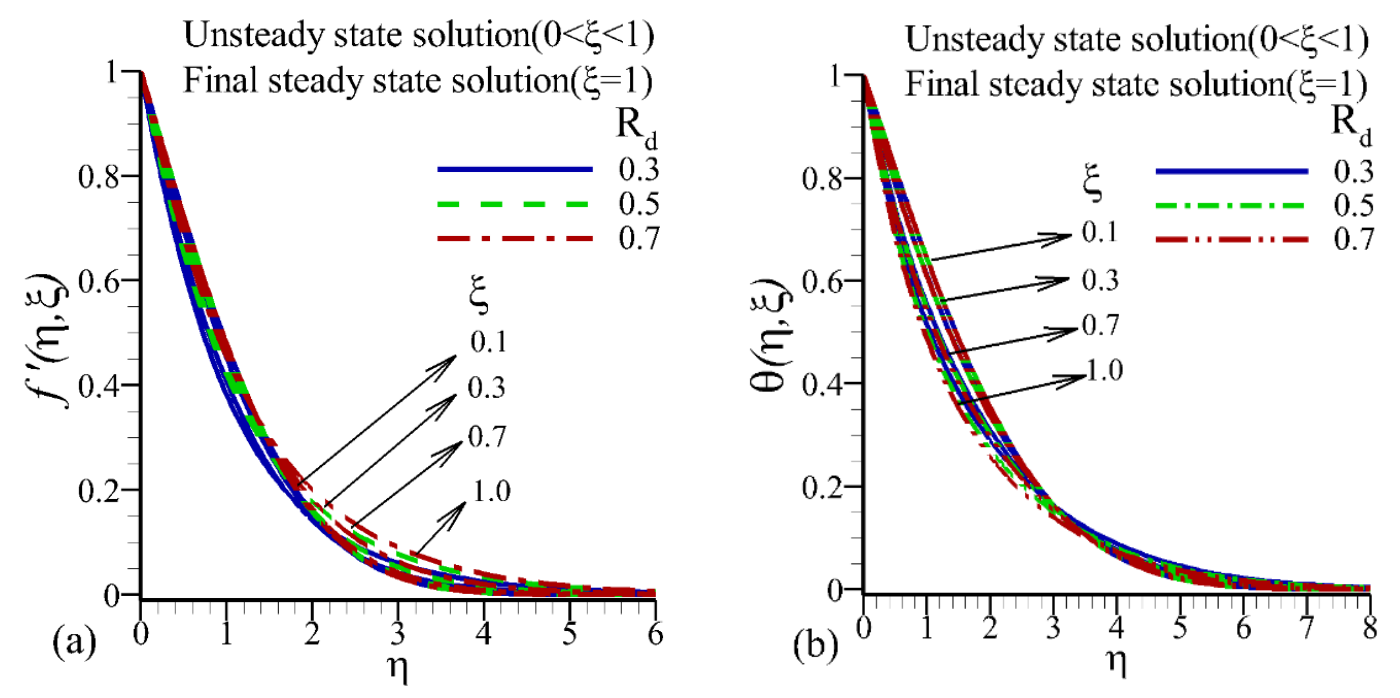

Figure 10. (a) Velocity $f^{\prime}(\eta, \xi),(\mathbf{b})$ Temperature $\theta(\eta, \xi)$ against $\eta$ for $R_{d}=0.3,0.5$, and $0.7, \xi=$ $0.1,0.3,0.7,1.0$, while $M=\beta=0.5$ and $\operatorname{Pr}=\lambda=1.0$.

\section{Concluding Remarks}

In the current study, a laminar two dimensional mixed convectional unsteady flow of a Maxwell fluid and heat transmission due to a continuous stretching sheet was analyzed under the effect of radiation and magnetic field. A series solution was given by employing finite difference technique, which relies on temperature profiles, skin friction, heat transfer, the magnetic field parameter $M$, radiation parameter $R_{d}$, Prandtl number $\operatorname{Pr}$, and relaxation time parameter $\beta$. It was observed that the solution was valid for the whole temporal $(0 \leq \tau<\infty)$ and spatial domain $(0 \leq \eta<\infty)$.

The major findings of our study are as under.

1. A decline in skin friction and the heat transfer rate was noted with a rise in values of the relaxation time parameter.

2. It was depicted that skin friction decreased with rising values of mixed convection parameter $\lambda$ and $\xi$, while the heat transmission rate showed the totally opposite behavior. 
3. The rise in radiation parameter $R_{d}$ reduced the local skin friction coefficient and led to enhance the heat transfer rate at the surface.

4. It was noticed that higher values of the Prandtl number Pr reduced the temperature and thermal boundary layer thickness rapidly.

5. A rise in velocity and decline in heat transfer rate were witnessed with lower Pr values.

6. The magnetic field parameter $M$ turned down the local skin friction.

7. The thermal diffusion rate could be controlled by varying the Prandtl number.

Author Contributions: S.H. and I.S.M. wrote the main manuscript text and performed mathematical modeling. Faraz and A.S.B. prepared the figures and tables. S.H., I.S.M., Y.-Z.L., Faraz and A.S.B. reviewed the manuscript. All authors have read and agreed to the published version of the manuscript.

Funding: This research was funded by National Natural Science Foundation of China, grant number 11271037.

Acknowledgments: We are grateful to Bushra Khan for the grammar and punctuation check.

Conflicts of Interest: The authors declare no conflict of interest.

\section{Nomenclature}

Pr Prandtl number

$q_{r} \quad$ Radiative heat flux

$C_{f x} \quad$ Skin friction coefficient

$N u_{x} \quad$ Local Nusselt number

$\operatorname{Re}_{\mathrm{x}} \quad$ Local Reynolds number

$c_{p} \quad$ Specific heat capacity

$G r_{x} \quad$ Local Grashof number

\section{Greek Symbols}

$\beta \quad$ Relaxation time parameter

$\rho \quad$ Fluid density

$\alpha \quad$ Thermal diffusivity

$k \quad$ Mean absorption coefficient

$\begin{array}{ll}B_{0} & \text { Magnetic field } \\ \mathrm{t} & \text { Time } \\ R_{d} & \text { Radiation parameter } \\ \mathrm{T} & \text { Temperature } \\ \mathrm{R}_{\mathrm{e}} & \text { Reynold number } \\ \mathrm{M} & \text { Magnetic field parameter } \\ v & \text { Kinematic viscosity }\end{array}$

$\sigma^{*} \quad$ Stefan-Boltzmann constant

$\psi \quad$ Stream function

$\tau_{w} \quad$ Wall shear stress

$\lambda \quad$ Mixed convection parameter

\section{References}

1. Ishak, A.; Nazar, R.; Pop, I. Hydro magnetic Flow and Heat Transfer Adjacent to a Stretching Vertical Sheet. Heat Mass Transf. 2008, 44, 921-927. [CrossRef]

2. Sakiadis, B.C. Boundary layer behavior on continuous solid surfaces. II: The boundary layer on a continuous flat surface. AICHE J. 1961, 7, 221-225. [CrossRef]

3. Crane, L.J. Flow Past a Stretching Plate. ZAMP 1970, 21, 645-647. [CrossRef]

4. Erickson, L.E.; Fan, L.T.; Fox, V.G. Heat and mass transfer on a moving continuous flat plate with suction or injection. Ind. Eng. Chem. Res. Fund. 1966, 5, 19-25. [CrossRef]

5. Gupta, P.S.; Gupta, A.S. Heat and mass transfer on a stretching sheet with suction or blowing. Can. J. Chem. Eng. 1977, 55, 744-746. [CrossRef]

6. Grubka, J.; Bobba, K.M. Heat transfer characteristics of a continuous stretching surface with variable temperature. ASME J. Heat Transf. 1985, 107, 248-250. [CrossRef]

7. Chen, C.K.; Char, M.I. Heat transfer of a continuous stretching surface with suction and injection. J. Math. Anal. Appl. 1988, 135, 568-580. [CrossRef]

8. Ali, M.E. Heat transfer characteristics of a continuous stretching surface. Heat Mass transf. 1994, 29, $227-234$. [CrossRef]

9. Chen, C.H. Laminar mixed convection adjacent to vertical continuous stretching sheets. Heat Mass transf. 1998, 33, 471-476. [CrossRef]

10. Wang, C.Y. Analysis of viscous flow due to stretching sheet with surface slip and suction. Nonlinear Anal. RWA 2009, 10, 375-380. [CrossRef] 
11. Andersson, H.I.; Bech, K.H.; Dandapat, B.S. Magneto hydrodynamic Flow of a Power Law Fluid over a Stretching Sheet. Int. J. Non Linear Mech. 1992, 27, 929-936. [CrossRef]

12. Hassanien, I.A. Flow and Heat Transfer on a Continuous Flat Surface Moving in a Parallel Free Stream of Power-Law Fluid. Appl. Model. 1996, 20, 779-784. [CrossRef]

13. Sadeghy, K.; Sharifi, M. Local Similarity Solution for the Flow of a 'Second-Grade' Viscoelastic Fluid above a Moving Plate. Int. J. Non Linear Mech. 2004, 39, 1265-1273. [CrossRef]

14. Serdar, B.; SalihDokuz, M. Three-Dimensional Stagnation Point Flow of a Second Grade Fluid Towards a Moving Plate. Int. J. Eng. Sci. 2006, 44, 49-58.

15. Haroun, M.H. Effect of Deborah Number and Phase Difference on Peristaltic Transport of a Third-Order Fluid in an Asymmetric Channel. Commun. Nonlinear Sci. Numer. Simul. 2007, 12, 1464-1480. [CrossRef]

16. Siddiqui, A.M.; Zeb, A.; Ghori, Q.K.; Benharbit, A.M. Homotopy Perturbation Method for Heat Transfer Flow of a Third Grade Fluid between Parallel Plates. Chaos Solitons Fractals 2008, 36, 182-192. [CrossRef]

17. Sajid, M.; Ahmad, I.; Hayat, T.; Ayub, M. Unsteady Flow and Heat Transfer of a Second Grade Fluid over a Stretching Sheet. Commun. Nonlinear Sci. Numer. Simul. 2009, 14, 96-108. [CrossRef]

18. Heyhat, M.M.; Khabazi, N. Non-Isothermal Flow of Maxwell Fluids above Fixed Flat Plates under the Influence of a Transverse Magnetic Field. Proc. Inst. Mech. Eng. C J. Mech. Eng. Sci. 2010, 225, 909-916. [CrossRef]

19. Kayvan, S.; Hajibeygi, H.; Taghavi, S.-M. Stagnation-point flow of upper-convected Maxwell fluids. Int. J. Non Linear Mech. 2006, 41, 1242-1247.

20. Aliakbar, V.; Alizadeh-Pahlavan, A.; Sadeghy, K. The influence of thermal radiation on MHD flow of Maxwellian fluids above stretching sheets. Commun. Nonlinear Sci. Numer. Simul. 2009, 14, 779-794. [CrossRef]

21. Alizadeh-Pahlavan, A.; Sadeghy, K. On the use of homotopy analysis method for solving unsteady MHD flow of Maxwellian fluids above impulsively stretching sheets. Commun. Nonlinear Sci. Numer. Simul. 2009, 14, 1355-1365. [CrossRef]

22. Hayat, T.; Abbas, Z.; Sajid, M. Series solution for the upper-convected Maxwell fluid over a porous stretching plate. Phys. Lett. A 2006, 358, 396-403. [CrossRef]

23. Abel, M.S.; Tawade, J.V.; Nandeppanavar, M.M. MHD flow and heat transfer for the upper-convected Maxwell fluid over a stretching sheet. Meccanica 2012, 47, 385-393.

24. Cess, R.D. The interaction of thermal radiation with free convection heat and mass transfer. Int. J. Heat Mass Transf. 1966, 9, 1269-1277. [CrossRef]

25. Hossain, M.A.; Takhar, H.S. Radiation effect on mixed convection along a vertical plate with uniform surface temperature. Heat Mass Transf. 1996, 31, 243-248. [CrossRef]

26. Cheng, E.H.; Özişik, M.N. Radiation with free convection in an absorbing, emitting and scattering medium. Int. J. Heat Mass Transf. 1972, 15, 1243-1252. [CrossRef]

27. Raptis, A. Radiation and free convection flow through a porous medium. Int. J. Commun. Heat Mass Transf. 1998, 25, 289-295. [CrossRef]

28. Hossain, M.A.; Alim, M.A.; Rees, D.A.S. The effect of radiation on free convection from a porous vertical plate. Int. J. Heat Mass Transf. 1999, 42, 181-191. [CrossRef]

29. Hayat, T.; Abbas, Z.; Pop, I.; Asghar, S. Effects of radiation and magnetic field on the mixed convection stagnation-point flow over a vertical stretching sheet in a porous medium. Int. J. Heat Mass Transf. 2010, 53, 466-474. [CrossRef]

30. Duwairi, H.M.; Duwairi, R.M. Thermal radiation effects on MHD-Rayleigh flow with constant surface heat flux. Heat Mass Transf. 2004, 41, 51-57. [CrossRef]

31. Gorla, R.S.R. Radiative effect on conjugate forced convection and conductive heat transfer in a circular pin. Int. J. Heat Fluid Flow 1988, 9, 49-51. [CrossRef]

32. Pop, S.R.; Grosan, T.; Pop, I. Radiation effects on the flow near the stagnation point of a stretching sheet. Tech. Mech. 2004, 25, 100-106.

33. Al-Odat, M.Q.; Damseh, R.A.; Al-Azab, T.A. Thermal boundary layer on an exponentially stretching continuous surface in the presence of magnetic field effect. Int. J. Appl. Mech. Eng. 2006, 11, 289-299.

34. Sajid, M.; Hayat, T. Influence of thermal radiation on the boundary layer flow due to an exponentially stretching sheet. Int. Commun. Heat Mass Transf. 2008, 35, 347-356. [CrossRef] 
35. Prasad, V.R.; Reddy, N.B.; Muthucumaraswamy, R. Radiation and mass transfer effects on two-dimensional flow past an impulsively started infinite vertical plate. Int. J. Therm. Sci. 2007, 46, 1251-1258. [CrossRef]

36. Andersson, H.I.; Aarseth, J.B.; Dandapat, B.S. Heat Transfer in a Liquid Film on an Unsteady Stretching Surface. Int. J. Heat Mass Transf. 2000, 43, 69-74. [CrossRef]

37. Elbashbeshy, E.M.A.; Bazid, M.A.A. Heat Transfer over an Unsteady Stretching Surface. Heat Mass Transf. 2004, 41, 1-4. [CrossRef]

38. Sharidan, S.; Mahmood, T.; Pop, I. Similarity Solutions for the Unsteady Boundary Layer Flow and Heat Transfer due to a Stretching Sheet. Int. J. Appl. Mech. Eng. 2006, 11, 647-654.

39. Ali, M.E.; Magyari, E. Unsteady Fluid and Heat Flow Induced by a Submerged Stretching Surface While Its Steady Motion is Slowed Down Gradually. Int. J. Heat Mass Transf. 2007, 50, 188-195. [CrossRef]

40. Dandapat, B.S.; Santra, B.; Vajravelu, K. The Effects of Variable Fluid Properties and Thermo-capillarity on the Flow of a Thin Film on an Unsteady Stretching Sheet. Int. J. Heat Mass Transf. 2007, 50, 991-996. [CrossRef]

41. Tsai, R.; Huang, K.H.; Huang, J.S. Flow and Heat Transfer over an Unsteady Stretching Surface with a Non-Uniform Heat Source. Int. Commun. Heat Mass Transf. 2008, 35, 1340-1343. [CrossRef]

42. Liu, I.C.; Andersson, H.I. Heat Transfer in a Liquid Film on an Unsteady Stretching Sheet. Int. J. Therm. Sci. 2008, 47, 766-772. [CrossRef]

43. Mukhopadhyay, S. Effect of Thermal Radiation on Unsteady Mixed Convection Flow and Heat Transfer over a Porous Stretching Surface in Porous Medium. Int. J. Heat Mass Transf. 2009, 52, 3261-3265. [CrossRef]

44. Mukhopadhyay, S. Effects of Slip on Unsteady Mixed Convective Flow and Heat Transfer Past a Stretching Surface. Chin. Phys. Lett. 2010, 27, 124401. [CrossRef]

45. Mukhopadhyay, S. Heat Transfer Analysis for Unsteady MHD Flow Past a Non-Isothermal Stretching Surface. Nucl. Eng. Des. 2011, 241, 4835-4839. [CrossRef]

46. Mukhopadhyay, S. Heat Transfer Analysis for Unsteady Flow of a Maxwell Fluid over a Stretching Surface in the Presence of a Heat Source/Sink. Chin. Phys. Lett. 2012, 29, 054703. [CrossRef]

47. Chamkha, A.J.; Aly, A.M.; Mansour, M.A. Similarity Solution for Unsteady Heat and Mass Transfer from a Stretching Surface Embedded in a Porous Medium with Suction/Injection and Chemical Reaction Effects. Chem. Eng. Commun. 2010, 197, 846-858. [CrossRef]

48. Bhattacharyya, K.; Mukhopadhyay, S.; Layek, G.C. Slip Effects on an Unsteady Boundary Layer Stagnation-Point Flow and Heat Transfer Towards a Stretching Sheet. Chin. Phys. Lett. 2011, 28, 094702. [CrossRef]

49. Ali, B.; Nie, Y.; Khan, S.A.; Sadiq, M.T.; Tariq, M. Finite element simulation of multiple slip effects on MHD unsteady Maxwell nanofluid flow over a permeable stretching sheet with radiation and thermo-diffusion in the presence of chemical reaction. Processes 2019, 7, 628. [CrossRef]

50. Na, T.Y. (Ed.) Computational Methods in Engineering Boundary Value Problems; Academic Press: New York, NY, USA, 1980.

(C) 2020 by the authors. Licensee MDPI, Basel, Switzerland. This article is an open access article distributed under the terms and conditions of the Creative Commons Attribution (CC BY) license (http://creativecommons.org/licenses/by/4.0/). 